\title{
Hematological parameters and peripheral blood morphologic abnormalities in children with COVID-19
}

\author{
Nese Yarali ${ }^{1}$, Yunus Akçabelen ${ }^{1}$, YASEMIN UNAL ${ }^{1}$, and Aslinur Ozkaya-Parlakay² \\ ${ }^{1}$ Ministry of Health Ankara City Hospital \\ ${ }^{2}$ Health Science University
}

May 28, 2020

\begin{abstract}
Objectives: The aim of this study is to evaluate the hematologic parameters and peripheral blood cell morphological changes in children with COVID-19 and compare them with those of children suspected but then confirmed to be negative for SARSCoV-2. Methods: Thirty children were tested to be positive for SARS-CoV-2 and the remaining 40 were negative. Hemoglobin, leukocyte, neutrophil, lymphocyte, monocyte counts according to age-specific intervals, platelet, large unstained cell counts, and delta neutrophil index were recorded. Differential counts were formulated by manual counting and morphology of the blood cells were evaluated. Results: The mean leukocyte counts of the SARS-CoV-2 positive and negative groups were 7.0 $\pm 3.7 \times 109 / \mathrm{L}$ and $10.4 \pm 7.1 \times 109 / \mathrm{L}$, respectively $(\mathrm{p}<0.05)$. Nine $(30 \%)$ children with COVID-19 had lymphopenia. Among children with COVID-19, absolute lymphocyte count was lower in those with pneumonia $(\mathrm{p}<0.05)$. Reactive lymphocytes were noted in $77.8 \%$ and $90 \%$ in the SARS-CoV-2 test positive and negative groups, respectively ( $>0.05)$. Mean absolute neutrophil counts of the SARS-CoV-2 test positive and negative groups were $3.7 \pm 2.9 \times 109 / \mathrm{L}$ and $5.4 \pm 4.2 \times 109 / \mathrm{L}(\mathrm{p}<0.05)$. Four patients (13.3\%) with SARS-CoV-2 test positive had neutrophilia and seven (23.3\%) had mild neutropenia. In the peripheral smear, vacuolated monocytes and dysplastic changes in neutrophils and platelets were noted in both groups. Conclusions: Leukocyte, neutrophil and monocyte counts were significantly lower in children with COVID-19 compared with symptomatic children without COVID-19. Lymphopenia, reactive lymphocytosis and dysplasia, could be noted in children with COVID-19. Further studies on hematological findings linked with the course of the disease in children are warranted.
\end{abstract}

\section{Introduction}

Coronavirus Disease 2019 (COVID-19) is an infectious disease with a rapid increase in cases and deaths since its first identification in Wuhan, China, in December 2019. The virus that causes COVID-19 is designated severe acute respiratory syndrome coronavirus 2 (SARS-CoV-2). Sore throat, high fever, shortness of breath, dry cough, headache, confusion, nausea, vomiting, diarrhea or loss of taste/smell are the most common manifestations of the disease $(1,2)$. COVID-19 testing includes either the detection of the virus itself by realtime reverse transcription polymerase chain reaction or those antibodies produced in response to infection (3). COVID-19 is predominantly prevalent among adults; patients under 18 years only account for $2 \%$ of the severely affected patients $(4,5)$.

Quantitative changes in blood cells, including leukocytes, lymphocytes, neutrophils, monocytes and platelets, have been reported in adults patients with COVID-19 (2, 6-8); moreover, the extent of such quantitative changes has been correlated with the severity of the disease in adults $(6,9)$. Normal (10), decreased (11) and increased (8) leukocyte counts have been reported. In general, decreased lymphocyte $(11,12)$ and increased neutrophil $(2,9,13)$, counts have been noted in various studies. However, there is paucity of data on the hematologic parameters of children with COVID-19 (4). In a review article on COVID-19 in children, a Chinese article was quoted reporting that "the most routine blood examinations were normal" (4). In another study reporting 10 pediatric patients affected with the disease, "a few cases" were reported to have 
leukopenia and lymphopenia (14). Moreover, although the morphological changes in peripheral blood cells have been reported in adults with COVID-19 (15), such changes, to our knowledge, have not been reported in children affected with the disease .

The aim of this retrospective study is to report the quantitative and qualitative changes of peripheral blood cells in children with COVID-19 and compare them with those of symptomatic children suspected but then confirmed to be negative for SARS-CoV-2.

\section{Methods}

Children admitted to the Emergency Department of Ministry of Health Ankara City Hospital Children's Hospital, which is a tertiary children hospital with 555 beds capacity, with symptoms including fever, sore throat, rhinorrhea, cough between 1-15 April 2020, were included in the study. All patients were tested for SARS-CoV-2 by quantitative real-time reverse transcription polymerase chain reaction from combined nasal and oropharyngeal swab samples. Complete blood count test and peripheral smear were done on admission of Emergency Department. Patients unable to obtain peripheral blood smear were excluded from the study. Thirty children were tested to be positive for SARS-CoV-2 and the remaining 40 symptomatic children were tested to be negative.

Complete blood count (CBC) was analyzed with Siemens ADVIA 2120i Hematology Analyzer with Auto slide (Siemens Healthcare Diagnostics, Erlangen, Germany); CBC results, including differential count results, were noted. Estimated absolute lymphocyte (ALC), neutrophil (ANC), monocyte (AMC) counts, were recorded from $\mathrm{CBC}$ results and were also calculated from peripheral smear specimens. Hemoglobin, leukocyte and neutrophil values were assessed according to age-specific intervals (16); ALC $<3 \times 10^{9} / \mathrm{L}$ and $\mathrm{ALC}<1.5 \times 10^{9} / \mathrm{L}$ were accepted as lymphopenia in $<12$-month and $>12$-month old patients, respectively (16). Neutropenia was diagnosed with an absolute neutrophil count of $<1.5 \times 10^{9} / \mathrm{L}(16)$. Neutrophil/lymphocyte ratio was calculated by the ADVIA 2120i analyzer. C-reactive protein values were also recorded.

All patients' peripheral blood smear specimens (May-Grünwald-Giemsa stain x100) were evaluated for neutrophil, thrombocyte and erythrocyte morphology and differential counts were formulated by manual counting including reactive lymphocyte and immature myeloid cells (band, metamyelocyte and myelocyte). the peripheral smears was blindly evaluated to the SARS-CoV-2 test result. The correlation between the reactive lymphocyte count in manual peripheral smear and large unstained cells (LUC), which reflects the activated lymphocytes and peroxidase-negative cells as measured by ADVIA 2120i, was analyzed. Moreover, the correlation between the percentage of immature myeloid cells in peripheral smear and delta neutrophil index (DNI) was assessed; DNI reflects immature myeloid cells and is calculated by subtracting polymorphonuclear leukocyte count in nuclear lobularity channel from neutrophil and eosinophil count in myeloperoxidase channel, both measured by the ADVIA 2120i analyzer.

The data were expressed as mean \pm SD. Student's t-test was used to compare the means. Pearson correlation was used to assess the relationship between two quantitative, continuous variables. A value of $\mathrm{p}<0.05$ was considered significant.

\section{Results}

The mean age of the whole cohort was $8.11 \pm 5$.71years (4 months-17 years). Thirty-five patients (50\%) were male; of the 30 SARS-CoV-2 test positive patients, $15(50 \%)$ were male. In the whole cohort $(\mathrm{n}=70)$, the most common presenting symptoms were fever $(65.7 \%)$ and cough $(60 \%)$. In the SARS-CoV-2 test positive and negative groups, 17 (56.7\%) and 19 negative patients (47.5\%) had pneumonia, respectively. None of the patients required treatment in the intensive care unit.

Complete blood count parameters of the SARS-CoV-2 test positive and negative groups were given in Table 1. Six patients (20\%) with SARS-CoV-2 test positive and 11 patients $(27.5 \%)$ with test negative were anemic according to age-specific intervals. Of note, a SARS-CoV-2 test positive 14-year-old Afghan patient with a diagnosis of aplastic anemia had pancytopenia before COVID-19. 
The mean leukocyte counts of the SARS-CoV-2 test positive and negative groups were $7.0 \pm 3.7 \times 10^{9} / \mathrm{L}$ and $10.4 \pm 7.1 \times 10^{9} / \mathrm{L}$, respectively $(\mathrm{p}<0.05)$. Within the SARS-CoV-2 test positive group, two patients $(6.7 \%)$ had leukocytosis and four patients $(13.3 \%)$ had leukopenia, according to age-specific intervals. The mean ALC of COVID-19 patients were $2.7 \pm 2.3 \times 10^{9} / \mathrm{L}$; nine $(30 \%)$ of them had lymphopenia, according to age-specific intervals. Twelve patients (30\%) in the SARS-CoV-2 test negative group had also lymphopenia. The ALCs of COVID-19 patients with and without pneumonia were $2.1 \pm 0.9 \times 10^{9} / \mathrm{L}$ and $3.4 \pm 2.9 \times 10^{9} / \mathrm{L}$, respectively $(\mathrm{p}<0.05)$. Reactive lymphocytes (Fig 1) were noted in $85.1 \%$ of the peripheral smears of the whole cohort; these figures were $77.8 \%$ and $90 \%$ in the SARS-CoV-2 test positive and negative groups. The mean absolute reactive lymphocyte (ARL) counts of SARS-CoV-2 test positive and negative groups were, $0.7 \pm 0.7 \times 10^{9} / \mathrm{L}$, $1.2 \pm 1.3 \times 10^{9} / \mathrm{L}$, respectively $(\mathrm{p}<0.05)$. The mean LUC counts of SARS-CoV-2 test positive and negative groups were $0.3 \pm 0.2 \times 10^{9} / \mathrm{L}$ and $0.2 \pm 0.23 \times 10^{9} / \mathrm{L}$, respectively $(\mathrm{p}>0.05)$. No correlation between LUC count and ARL count was noted. The respective mean absolute neutrophil counts were $3.7 \pm 2.9 \times 10^{9} / \mathrm{L}$ and $5.4 \pm 4.2$ $\mathrm{x} 10^{9} / \mathrm{L}(\mathrm{p}<0.05)$. Four patients $(13.3 \%)$ with SARS-CoV-2 test positive and 10 patients $(25 \%)$ with negative result groups had neutrophilia. Seven (23.3\%) COVID-19 patients had mild neutropenia, whereas $3(7.5 \%)$ patients with test negative had neutropenia. The mean neutrophil/lymphocyte ratios of SARS-CoV-2 positive and negative groups were $2.7 \pm 2.9$ and $2.9 \pm 4.2$, respectively ( $\mathrm{p}>0.05)$. Immature myeloid cells $(>2 \%$ in blood smear) were noted in 13 children (23\%) in the COVID-19 group. All COVID-19 patients' DNI were $<0.4$. The mean AMC was $0.4 \pm 0.3 \times 10^{9} / \mathrm{L}$ in the SARS-CoV-2 positive and $0.6 \pm 0.5 \times 10^{9} / \mathrm{L}$ in the SARS-CoV-2 negative patients $(\mathrm{p}<0.05)$. Leukoerythroblastic reaction was not noted in any children in the whole cohort.

The mean platelet count was $268 \pm 89 \times 10^{9} / \mathrm{L}$ in the SARS-CoV-2 positive and $339 \pm 178 \times 10^{9} / \mathrm{L}$ in the SARSCoV-2 negative group $(\mathrm{p}<0.05)$ (Table 1$)$. In the whole cohort, thrombocytopenia was detected in four patients; one of them was the patient with aplastic anemia and COVID-19; the remaining three were in the SARS-CoV-2 negative group. Thrombocytosis was noted in one patient only. The mean platelet volume (MPV) was comparable between the two groups; with no child noted to have increased MPV. The mean C-reactive protein level of the SARS-CoV-2 positive group was significantly lower when compared with that of SARS-CoV-2 negative group ( $\mathrm{p}=0.006)$.

In the peripheral smears of a few children affected by COVID-19, vacuolated monocytes $(\mathrm{n}=3 ; 13.3 \%)$ and dysplastic changes such as hypergranulation/lobulation abnormalities in neutrophils $(\mathrm{n}=11 ; 36.7 \%)$ were noted (Figs. 2, and 3). The respective figures within the non-COVID-19 group were 4 (10.0\%) and $9(22.5 \%)$. Although the MPV values were within the normal range, $20 \%$ of the COVID-19 patients had giant platelets (Figure 4); however, giant platelets were also noted in $27.5 \%$ of the negative patients.

\section{Discussion}

In adults, the most common hematological findings of COVID-19 include lymphocytopenia $(11,12)$, neutrophilia $(2,13)$, mild thrombocytopenia $(7)$ and, less frequently, thrombocytosis $(9,17)$. However, there is paucity of data on hematological findings in affected children $(4,13)$. In our study, the majority of the SARSCoV-2 infected children had normal leukocyte count, but $13.3 \%$ had leukopenia and $6.7 \%$ had leukocytosis, according to age-specific intervals. Despite normal leukocyte count, lymphopenia and neutrophilia were noted in $30.0 \%$ and $13.3 \%$ of children affected with the disease, respectively. Seven patients with SARS-CoV-2 test positive $(23.3 \%)$ had neutropenia; this figure was $7.5 \%$ in the test negative group. Neutropenia has not been previously reported in adults affected with the disease.

The presence of reactive lymphocytes has been occasionally reported in adults with COVID-19. Fan et al. reported a few lymphoplasmacytoid reactive lymphocytes in peripheral blood of lymphopenic patients with COVID-19 (7). It is well documented in the literature that in response to stress, atypical reactive lymphocytes that are characterized by nuclear and cytoplasmic distortion appear in the blood (18). Reactive lymphocytes were detected in $85.1 \%$ of our patients in the whole cohort, $77.8 \%$ in the test-positive and $90 \%$ in the test-negative groups. The mean ARL count was lower in the COVID-19 positive group, which was statistically different from the COVID-19 negative group. Leukoerythroblastic reaction that reflects the immature erythroid and immature myeloid cells circulating in the peripheral blood has also been reported in adults with COVID-19 (19). However, we did not observe leukoerythroblastosis in the whole cohort, but 
we noted bands and metamyelocytes in $23 \%$ of the COVID-19 patients' peripheral smear. We detected the lack of concordance between LUC/DNI as measured by the counter and manual counting. This addresses the importance to assess peripheral blood smears.

Thrombocytopenia was detected in only one patient with aplastic anemia and COVID-19; thrombocytosis was not noted in any patient. In a meta-analysis, low platelet count has been associated with the increased severity of the disease and increased mortality in adults with COVID-19, thus serving as an indicator of worsening illness during hospitalization (9). The absence of thrombocytopenia in our series may be related to better clinical prognosis of the disease in children.

Zini et al. from Italy reported marked morphological abnormalities in neutrophil lineage and platelet morphology in adults with COVID-19, mainly very large, usually hyperchromatic platelets, both in patients with thrombocytosis and thrombocytopenia (15). We noted some nonspecific dysplastic changes in peripheral smear of affected children. Giant platelets were noted in $20 \%$ of children with COVID-19. Vacuolated monocytes, hypergranulated neutrophils and pseudo Pelger-Huet abnormality were also seen in 13\%, $7 \%$ and $30 \%$ of COVID-19 infected children, respectively. In patients with COVID-19, upregulation of pro-inflammatory cytokines in the blood, including interleukin (IL)-1, IL-6, TNF, and interferon $\gamma$ has been reported (5). Dysregulation of immunological environment may have an important role in the pathogenesis of myelodysplastic syndromes (20). We do not have the data for pro-inflammatory cytokines in the blood and we speculate that the dysplastic changes of blood cells in our series might be related to those altered cytokines.

In conclusion, leukocyte and neutrophil counts were lower in children with COVID-19 compared with children with similar symptoms. Lymphopenia and reactive lymphocytosis, dysplastic changes of granulocytic lineage and giant platelets on peripheral smear, although not specific, could be noted in children with COVID-19. Further studies on hematological findings linked with the course of the disease in children are warranted.

Conflict of Interest: The authors have indicated they have no potential conflicts of interest to disclose.

\section{REFERENCES}

1. Hui DS, Azhar IE, Madani TA, et al. The continuing 2019-nCoV epidemic threat of novel coronaviruses to global health - The latest 2019 novel coronavirus outbreak in Wuhan, China. Int J Infect Dis . 2020;91:264-6.

2. Chen N, Zhou M, Dong X, et al. Epidemiological and clinical characteristics of 99 cases of 2019 novel coronavirus pneumonia in Wuhan, China: a descriptive study. Lancet.2020;395(10223):507-13.

3. Huang C, Wang Y, Li X, et al. Clinical features of patients infected with 2019 novel coronavirus in Wuhan, China. Lancet.2020;395(10223):497-506.

4. She J, Liu L, Liu W. COVID-19 epidemic: Disease characteristics in children. J Med Virol. 2020. Mar 31.doi: $10.1002 / \mathrm{jmv} .25807$

5. Molloy EJ, Bearer CF. COVID-19 in children and altered inflammatory responses. Pediatr Res. 2020. doi: 10.1038/s41390-020-0881-y

6. Yuen KS, Ye ZW, Fung SY, et al. SARS-CoV-2 and COVID-19: The most important research questions. Cell Biosci . 2020;10:40.

7. Fan BE, Chong VCL, Chan SSW, et al. Hematologic parameters in patients with COVID-19 infection. Am J Hematol. 2020. doi: 10.1002/ajh.25774

8. Henry BM, de Oliveira MHS, Benoit S, et al. Hematologic, biochemical and immune biomarker abnormalities associated with severe illness and mortality in coronavirus disease 2019 (COVID-19): a metaanalysis. Clin Chem Lab Med . 2020. doi: 10.1515/cclm-2020-0369

9. Lippi G, Plebani M, Henry BM. Thrombocytopenia is associated with severe coronavirus disease 2019 (COVID-19) infections: A meta-analysis. Clin Chim Acta . 2020;506:145-8. 
10. Zhang JJ, Dong X, Cao YY, et al. Clinical characteristics of 140 patients infected with SARS-CoV-2 in Wuhan, China. Allergy . 2020. doi: 10.1111/all.14238

11. Guan WJ, Ni ZY, Hu Y, et al. Clinical Characteristics of Coronavirus Disease 2019 in China. N Engl J Med. 2020. doi: 10.1056/NEJMoa2002032

12. Wang F, Nie J, Wang H, et al. Characteristics of peripheral lymphocyte subset alteration in COVID-19 pneumonia. J Infect Dis . 2020. doi: 10.1093/infdis/jiaa150

13. Mo P, Xing Y, Xiao Y, et al. Clinical characteristics of refractory COVID-19 pneumonia in Wuhan, China. Clin Infect Dis . 2020. doi: 10.1093/cid/ciaa270

14. Xu Y, Li X, Zhu B, et al. Characteristics of pediatric SARS-CoV-2 infection and potential evidence for persistent fecal viral shedding. Nat Med . 2020; 26:502-5.

15. Zini G, Bellesi S, Ramundo F, et al. Morphological anomalies of circulating blood cells in COVID-19. Am J Hematol. 2020. doi: 10.1002/ajh.25824

16. Walkovich KL, Newburger PE. Leukopenia. In: Kliegman RM, Stanton BF, St Geme III JW, Schor NF, Behrman RE eds. Nelson Textbook of Pediatrics , 20th ed. International Edition: Elsevier; 2016: 1047-1055.

17. Ruan Q, Yang K, Wang W, et al. Clinical predictors of mortality due to COVID-19 based on an analysis of data of 150 patients from Wuhan, China. Intensive Care Med. 2020. doi: 10.1007/s00134-020-05991-x

18. Barbara J. Bain BJ. Blood Cells . $5^{\text {th }}$ ed. Wiley Blackwell; 2015.

19. Mitra A, Dwyre DM, Schivo M, et al. Leukoerythroblastic reaction in a patient with COVID-19 infection. Am J Hematol. 2020. doi: 10.1002/ajh.25793

20. Shi X, Zheng Y, Xu L, Cao C, Dong B, Chen X. The inflammatory cytokine profile of myelodysplastic syndromes: A meta-analysis.Medicine (Baltimore) . 2019;98:e15844.

Figure Legends:

1: Reactive lymphocytes (x100) 2: Lobulation abnormalities (x100) 3: Hypergranulation and hypolobulation in a neutrophil (x100) 4: Giant platelet and platelet anisocytosis (x100)

\section{Hosted file}

Table 1 covid -19.docx available at https://authorea.com/users/320139/articles/454868hematological-parameters-and-peripheral-blood-morphologic-abnormalities-in-childrenwith-covid-19 

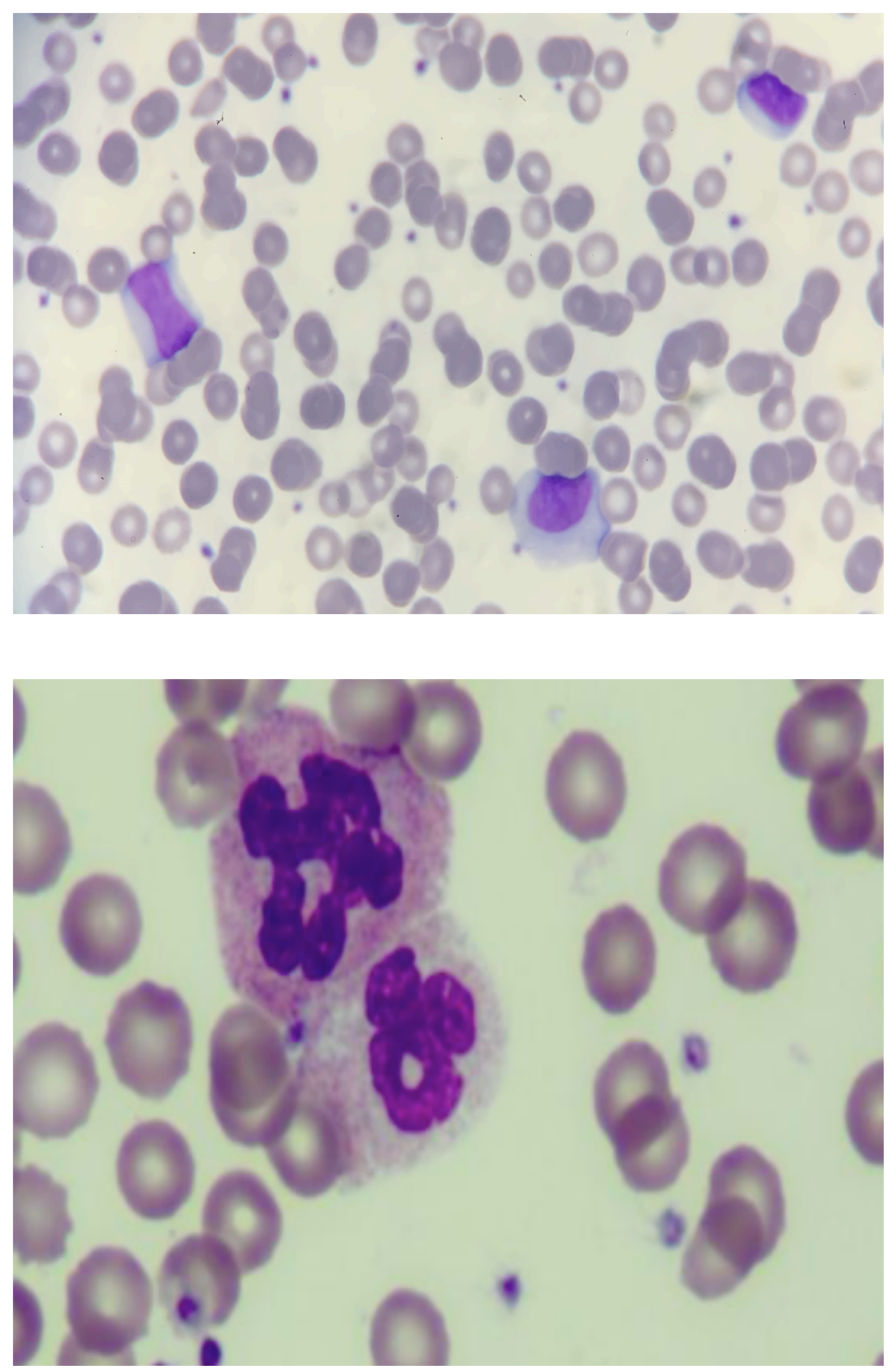

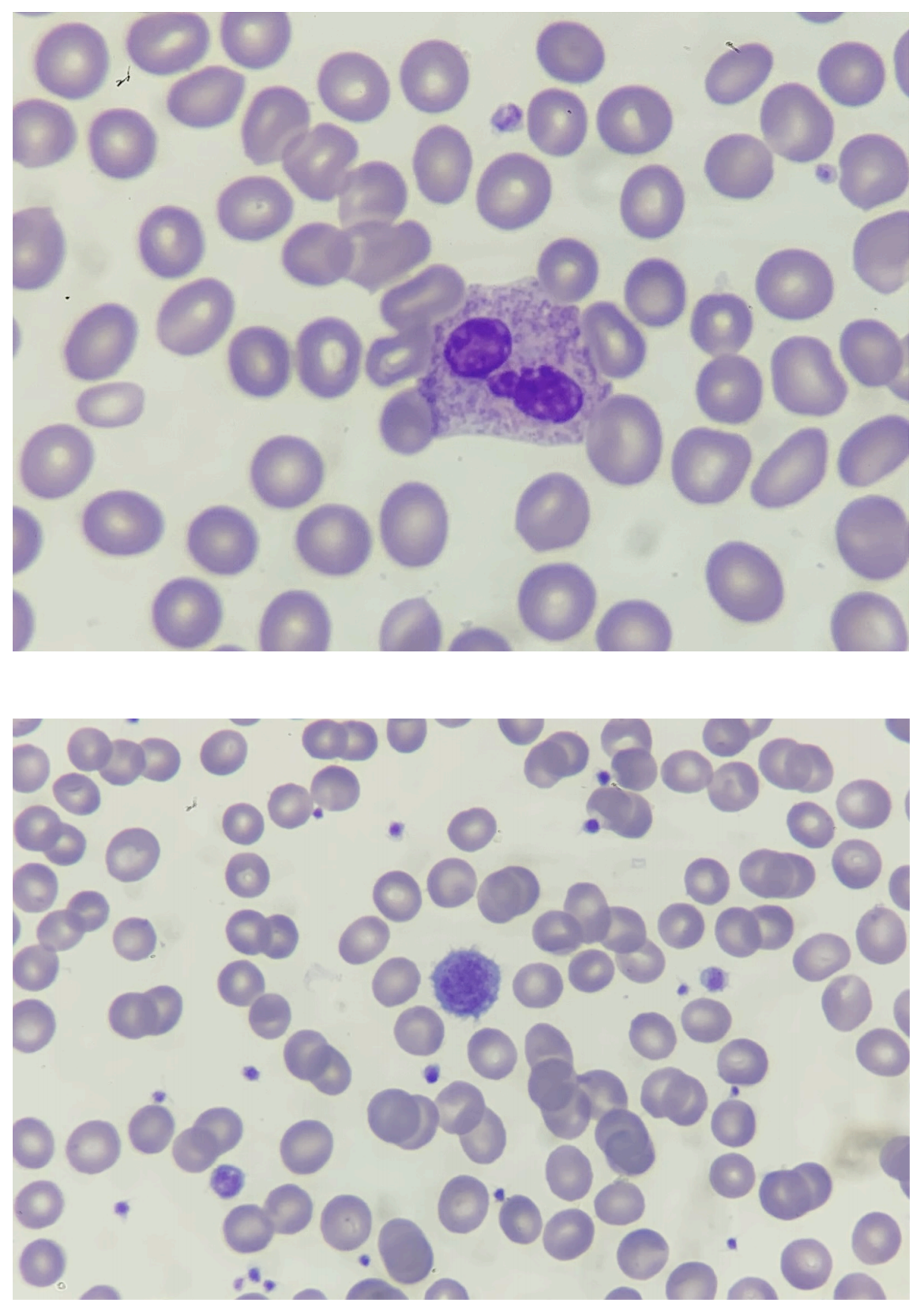